\title{
Focus on JNJ-Q2, a novel fluoroquinolone, for the management of community-acquired bacterial pneumonia and acute bacterial skin and skin structure infections
}

\author{
This article was published in the following Dove Press journal: \\ Infection and Drug Resistance \\ 7 June 2016 \\ Number of times this article has been viewed
}

\author{
Travis $M$ Jones ${ }^{1,2}$ \\ Steven W Johnson ${ }^{1,3}$ \\ $\checkmark$ Paul DiMondi ${ }^{1,4}$ \\ Dustin TWilson ${ }^{1,2}$ \\ 'Department of Pharmacy Practice, \\ College of Pharmacy and Health \\ Sciences, Campbell University, Buies \\ Creek, ${ }^{2}$ Department of Pharmacy, \\ Duke University Hospital, Durham, \\ ${ }^{3}$ Department of Pharmacy, Forsyth \\ Medical Center, Novant Health, \\ Winston-Salem, ${ }^{4}$ Department of \\ Pharmacy, Durham VA Medical Center, \\ Durham, NC, USA
}

\begin{abstract}
JNJ-Q2 is a novel, fifth-generation fluoroquinolone that has excellent in vitro and in vivo activity against a variety of Gram-positive and Gram-negative organisms. In vitro studies indicate that JNJ-Q2 has potent activity against pathogens responsible for acute bacterial skin and skin structure infections (ABSSSI) and community-acquired bacterial pneumonia (CABP), such as Staphylococcus aureus and Streptococcus pneumoniae. JNJ-Q2 also has been shown to have a higher barrier to resistance compared to other agents in the class and it remains highly active against drug-resistant organisms, including methicillin-resistant $S$. aureus, ciprofloxacinresistant methicillin-resistant $S$. aureus, and drug-resistant $S$. pneumoniae. In two Phase II studies, the efficacy of JNJ-Q2 was comparable to linezolid for ABSSSI and moxifloxacin for CABP. Furthermore, JNJ-Q2 was well tolerated, with adverse event rates similar to or less than other fluoroquinolones. With an expanded spectrum of activity and low potential for resistance, JNJ-Q2 shows promise as an effective treatment option for ABSSSI and CABP. Considering its early stage of development, the definitive role of JNJ-Q2 against these infections and its safety profile will be determined in future Phase III studies.
\end{abstract}

Keywords: JNJ-Q2, fluoroquinolone, ABSSSI, CABP, MRSA

\section{Introduction}

The emergence of antibiotic resistance among Gram-positive and Gram-negative bacteria poses a serious public health threat and warrants immediate action., ${ }^{1,2}$ Each year, more than two million people in the US are infected by antibiotic-resistant bacteria. ${ }^{3}$ Antibiotic resistance diminishes the antibiotic armamentarium available to health care providers; therefore, patients often receive antibiotics that may be more toxic, more expensive, and less effective than the standard of care. ${ }^{4}$ To combat this threat, the development of new antibiotics has become a major initiative of the Centers for Disease Control and Prevention (CDC).

Fluoroquinolones are potent, broad-spectrum antibacterial agents that have been mainstays of therapy for numerous community-acquired and hospital-acquired infections since the late $1980 \mathrm{~s} .{ }^{4}$ Over the years, however, extensive use of the more commonly prescribed agents (ie, ciprofloxacin, levofloxacin, and moxifloxacin) has led to the evolution of fluoroquinolone-resistant bacteria, limiting their clinical use. ${ }^{5}$ Shortly after ciprofloxacin was made commercially available, resistance to this agent emerged among methicillin-susceptible Staphylococcus aureus (MSSA) and methicillin-resistant
Correspondence: Travis M Jones

Department of Pharmacy,

Duke University Hospital, PO Box 3089,

Durham, NC 27710, USA

$\mathrm{Tel}+\mathrm{I} 9196681956$

$\mathrm{Fax}+\mathrm{I} 9196812741$

Email tjones@campbell.edu
Infection and Drug Resistance 2016:9 119-128

(c) (1) (5) 2016 Jones et al. This work is published and licensed by Dove Medical Press Limited. The full terms of this license are avalable at https://www.dovepress.com/terms. cc) php and incorporate the Creative Commons Attribution - Non Commercial (unported, v3.0) License (http://creativecommons.org/licenses/by-nc/3.0/). By accessing the work you hereby accept the Terms. Non-commercial uses of the work are permitted without any further permission from Dove Medical Press Limited, provided the work is properly attributed. For permission for commercial use of this work, please see paragraphs 4.2 and 5 of our Terms (https.//www.dovepress.com/terms.php).
Dovepress

http://dx.doi.org/1 0.2147/IDR.S105620
119 
S. aureus (MRSA). ${ }^{6,7}$ Furthermore, US surveillance data from 2008 showed that $70 \%$ of MRSA isolates from complicated skin and skin structure infections were resistant to levofloxacin. ${ }^{8}$ Given their insufficient activity against MRSA, no US Food and Drug Administration (FDA)-approved fluoroquinolone is recommended for treatment of MRSArelated infections. ${ }^{5}$ Due to the predominance of MRSA in acute bacterial skin and skin structure infections (ABSSSI), fluoroquinolones are no longer recommended as empiric therapy. ${ }^{9}$ Respiratory fluoroquinolones, including levofloxacin and moxifloxacin, have been important therapeutic options for community-acquired bacterial pneumonia (CABP) due to their enhanced activity against Streptococcus pneumoniae, compared to ciprofloxacin. ${ }^{10}$ Studies ${ }^{11,12}$ have shown that levofloxacin and moxifloxacin resistance in S. pneumoniae isolates have remained rare in the US $(<2 \%)$. Although the incidence of fluoroquinolone resistance in $S$. pneumoniae is low, the rising rates of resistance to other therapies (eg, penicillins, cephalosporins, and macrolides) is concerning enough to question whether resistance will eventually be seen with levofloxacin and moxifloxacin. ${ }^{13}$ A novel fluoroquinolone with potent activity against MRSA and drug-resistant $S$. pneumoniae (DRSP) could prove to be an invaluable treatment option for ABSSSI and CABP in the future.

JNJ-Q2 (also known as avarofloxacin; Allergan, Parsippany, NJ, USA) is a novel, fifth-generation fluoroquinolone with antimicrobial activity against resistant Gram-positive and Gram-negative organisms. JNJ-Q2 displays potent in vitro activity against DRSP and $S$. aureus isolates, including MRSA and fluoroquinolone-resistant MRSA isolates. ${ }^{6,14,15}$ Phase III clinical trials are awaited, however, in February 2013, JNJ-Q2 was designated a qualified infectious disease product, therefore, it will be eligible for fast-track designation and priority review from the FDA. ${ }^{16}$ The primary aim of this review is to describe the characteristics of this investigational fluoroquinolone and to evaluate its potential place in therapy for ABSSSI and CABP.

\section{Pharmacology}

\section{Chemical structure}

JNJ-Q2 belongs to the series of aminoethylidenylpiperidine fluoroquinolones. ${ }^{14,17}$ Similar to other fluoroquinolones, JNJ-Q2 shares a structural feature known as the quinolone nucleus (Figure 1). ${ }^{18}$ The evolution of fluoroquinolones is attributed to modifications of the quinolone nucleus through additions of various substituents at the N-1, C-6, C-7, and C-8 positions. ${ }^{18-20}$ Modifications at these sites are associated with altered antimicrobial activity, interaction with their

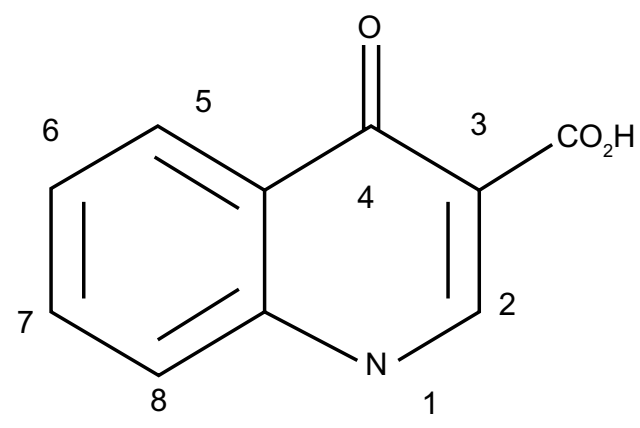

Figure I Common structural features of quinolones.

pharmacological target, pharmacokinetics, and adverse effect profile. ${ }^{16,20}$ Consistent with other fluoroquinolones, JNJ-Q2 has a fluorine atom at position $\mathrm{C}-6$, the addition of a methoxy group at C-8 (gatifloxacin and moxifloxacin), and the addition of a cyclopropyl group at N-1 (gatifloxacin, gemifloxacin, and moxifloxacin). However, unique to JNJ-Q2 is the modification at the $\mathrm{C}-7$ position (Figure 2). The chemical properties of JNJ-Q2 include low molecular weight and satisfactory solubility and lipophilicity. ${ }^{14}$ These characteristics are suggestive of absorption and permeability comparable to those of currently available fluoroquinolones. ${ }^{14}$

\section{Mechanism of action}

JNJ-Q2 directly inhibits bacterial DNA synthesis by halting the activity of DNA gyrase (responsible for negative helical supercoiling) and DNA topoisomerase IV (responsible for separating the nucleotide strands). Most fluoroquinolones have an affinity for one of these enzymes. However, JNJ-Q2 offers improved balance in the potencies of inhibition of both DNA topoisomerase targets, which is thought to decrease the development of resistance. ${ }^{14,15,21}$ This balance in potency may be attributed to the modification of the methoxy group addition at the $\mathrm{C}-8$ position. ${ }^{22}$

\section{Microbiology}

JNJ-Q2 displayed excellent in vitro and in vivo activity against several Gram-negative and Gram-positive organisms,

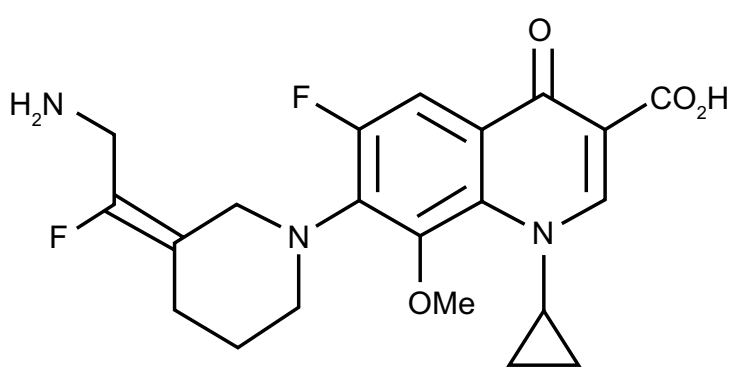

Figure 2 Chemical structure of JNJ-Q2. 
including MSSA, MRSA, ciprofloxacin-resistant MRSA, and fluoroquinolone-resistant $S$. pneumoniae..$^{6,14,15,17,23-25}$ The in vitro activity of JNJ-Q2 is summarized in Table 1.

\section{In vitro activity}

\section{Potency against $S$. aureus}

The in vitro potency of JNJ-Q2 against MRSA, MSSA, and ciprofloxacin-resistant $S$. aureus strains was compared to those of moxifloxacin, ciprofloxacin, and vancomycin. JNJ-Q2 was the most potent antimicrobial against MRSA with a minimum inhibitory concentration for $90 \%$ of isolates $\left(\mathrm{MIC}_{90}\right.$ ) of $0.25 \mu \mathrm{g} / \mathrm{mL}$ compared to $>32 \mu \mathrm{g} / \mathrm{mL}, 8 \mu \mathrm{g} / \mathrm{mL}$, and $1 \mu \mathrm{g} / \mathrm{mL}$ for ciprofloxacin, moxifloxacin, and vancomycin, respectively. Furthermore, ciprofloxacin-resistant

Table I In vitro activity of JNJ-Q2

\begin{tabular}{|c|c|c|}
\hline Organism & $\begin{array}{l}\text { Number of } \\
\text { isolates }\end{array}$ & $\begin{array}{l}\text { MIC }_{90} \\
(\mu \mathrm{g} / \mathrm{mL})\end{array}$ \\
\hline S. pneumoniae, all isolates ${ }^{6,14}$ & 2,255 & $0.015-0.12$ \\
\hline S. pneumoniae, ciprofloxacin resistant ${ }^{14}$ & 19 & 0.25 \\
\hline S. aureus, all isolates ${ }^{14}$ & 406 & 0.25 \\
\hline $\mathrm{MSSA}^{14}$ & 26 & 0.015 \\
\hline MRSA $^{14}$ & 345 & 0.25 \\
\hline MRSA, ciprofloxacin resistant ${ }^{14}$ & 281 & $0.25-0.5$ \\
\hline MSSE $^{14}$ & 30 & 0.015 \\
\hline MRSE, all isolates ${ }^{14}$ & 34 & 0.25 \\
\hline MRSE, ciprofloxacin resistant ${ }^{14}$ & 21 & 0.25 \\
\hline S. pyogenes ${ }^{14}$ & 21 & 0.015 \\
\hline S. agalactiae ${ }^{14}$ & 19 & 0.015 \\
\hline Streptococcus spp., group $C^{14}$ & 10 & 0.015 \\
\hline E. faecalis ${ }^{14}$ & 12 & 0.5 \\
\hline E. faecium ${ }^{14}$ & 13 & 4 \\
\hline H. influenzae 6,14 & $\mathrm{I}, 200$ & 0.015 \\
\hline M. catarrhalis ${ }^{6,14}$ & 455 & $\leq 0.015$ \\
\hline E. coli, all isolates ${ }^{14}$ & 30 & 0.25 \\
\hline E. coli, ciprofloxacin resistant ${ }^{14}$ & 20 & 16 \\
\hline E. cloacae ${ }^{14}$ & 26 & 0.12 \\
\hline E. aerogenes ${ }^{14}$ & 26 & 0.25 \\
\hline K. pneumoniae ${ }^{14}$ & 27 & 0.25 \\
\hline S. marcescens ${ }^{14}$ & 13 & I \\
\hline P. mirabilis ${ }^{14}$ & II & 0.5 \\
\hline P. stuartiil ${ }^{14}$ & 10 & 4 \\
\hline C. freundii ${ }^{14}$ & 13 & 8 \\
\hline P. aeruginosa $a^{14}$ & 28 & 2 \\
\hline N. gonorrhoeae, all isolates ${ }^{42}$ & 75 & 0.25 \\
\hline N. gonorrhoeae, ciprofloxacin resistant ${ }^{42}$ & 31 & 0.25 \\
\hline
\end{tabular}

Abbreviations: $\mathrm{MIC}_{90}$, minimum inhibitory concentration required to inhibit the growth of $90 \%$ of organisms; S. pneumoniae, Streptococcus pneumoniae; S. aureus, Staphylococcus aureus; MSSA, methicillin-susceptible S. aureus; MRSA, methicillinresistant S. aureus; MSSE, methicillin-susceptible Staphylococcus epidermidis; MRSE, methicillin-resistant S. epidermidis; S. pyogenes, Streptococcus pyogenes; S. agalactiae, Streptococcus agalactiae; E. faecalis, Enterococcus faecalis; E. faecium, Enterococcus faecium; H. influenzae, Haemophilus influenzae; M. catarrhalis, Moraxella catarrhalis; E. coli, Escherichia coli; E. cloacae, Enterobacter cloacae; E. aerogenes, Enterobacter aerogenes; K. pneumoniae, Klebsiella pneumoniae; S. marcescens, Serratia marcescens; P. mirabilis, Proteus mirabilis; $P$. stuartii, Providencia stuartii; $C$. freundii, Citrobacter freundii; $P$. aeruginosa, Pseudomonas aeruginosa; $N$. gonorrhoeae, Neisseria gonorrhoeae.
MRSA strains remained susceptible to JNJ-Q2, with $\mathrm{MIC}_{90}$ of $0.25 \mu \mathrm{g} / \mathrm{mL}$, compared to $68 \mu \mathrm{g} / \mathrm{mL}, 8 \mu \mathrm{g} / \mathrm{mL}$, and $1 \mu \mathrm{g} / \mathrm{mL}$ for ciprofloxacin, moxifloxacin, and vancomycin, respectively. ${ }^{14}$ For MSSA isolates, JNJ-Q2 continues to have the lowest $\mathrm{MIC}_{90}(0.015 \mu \mathrm{g} / \mathrm{mL})$ compared to ciprofloxacin $(0.5 \mu \mathrm{g} / \mathrm{mL})$, moxifloxacin $(0.12 \mu \mathrm{g} / \mathrm{mL})$, and vancomycin $(2 \mu \mathrm{g} / \mathrm{mL}) .{ }^{14}$

In 2010, another in vitro study ${ }^{23}$ evaluated JNJ-Q2's activity against 3,081 isolates of $S$. aureus (MRSA and MSSA) collected from several countries around the world. JNJ-Q2 had an associated $\mathrm{MIC}_{90}$ of $0.5 \mu \mathrm{g} / \mathrm{mL}$ for all isolates, including levofloxacin-resistant and -susceptible MRSA and MSSA. The $\mathrm{MIC}_{90}$ was similar to those of trimethoprimsulfamethoxazole $(\leq 0.5 \mu \mathrm{g} / \mathrm{mL})$ and daptomycin $(0.5 \mu \mathrm{g} / \mathrm{mL})$, as well as being lower than all other comparators, including other fluoroquinolones, linezolid, clindamycin, oxacillin, erythromycin, tetracycline, and vancomycin. ${ }^{23}$

Lastly, an in vitro study ${ }^{15}$ evaluated the potency of JNJQ2 against $S$. aureus with various fluoroquinolone-resistant target mutations. In this study, JNJ-Q2 was the most active agent compared to clinafloxacin, nadifloxacin, moxifloxacin, levofloxacin, and ciprofloxacin. JNJ-Q2 had associated MIC values that were 16- to 1,024-fold lower than those of the other agents. Furthermore, mutations upregulating efflux pumps (eg, NorA), alone or in combination with target site mutations, did not change the MIC values for JNJ-Q2. ${ }^{15}$

\section{Potency against beta-hemolytic streptococci}

In an in vitro study ${ }^{14}$ comparing common skin pathogens, JNJ-Q2 was the most potent agent against beta-hemolytic streptococci compared to moxifloxacin and ciprofloxacin. JNJ-Q2 had $\mathrm{MIC}_{90}$ of $0.015 \mu \mathrm{g} / \mathrm{mL}$ for $S$. pyogenes, $S$. agalactiae, and Streptococcus spp. group C, compared to $0.25 \mu \mathrm{g} / \mathrm{mL}$ and $1 \mu \mathrm{g} / \mathrm{mL}$ for moxifloxacin and ciprofloxacin, respectively. ${ }^{14}$ The findings of another in vitro study ${ }^{23}$ confirmed the activity of JNJ-Q2 against streptococcal spp., with $\mathrm{MIC}_{90}$ values of $0.015 \mu \mathrm{g} / \mathrm{mL}$ for 278 isolates of $S$. pyogenes and 161 isolates of $S$. agalactiae. Similar to the aforementioned study, ${ }^{14} \mathrm{JNJ}-\mathrm{Q} 2$ was the most potent agent against beta-hemolytic streptococci compared to moxifloxacin, levofloxacin, and ciprofloxacin..$^{23}$

\section{Potency against S. pneumoniae}

In an in vitro study ${ }^{14}$ evaluating skin and respiratory tract pathogens, JNJ-Q2 demonstrated the most potent activity against $S$. pneumoniae isolates compared to all other agents tested (moxifloxacin, gemifloxacin, levofloxacin, ciprofloxacin, penicillin, and erythromycin), including isolates 
classified as fluoroquinolone-resistant ones. JNJ-Q2 had a $\mathrm{MIC}_{90}$ value of $0.12 \mu \mathrm{g} / \mathrm{mL}$ against 118 isolates of $S$. pneumoniae, and this was associated with a fourfold and 32 -fold increase in potency compared to gemifloxacin and moxifloxacin, respectively. Furthermore, the JNJ-Q2 $\mathrm{MIC}_{90}$ was $0.25 \mu \mathrm{g} / \mathrm{mL}$ against 19 isolates of ciprofloxacin- and levofloxacin-resistant clinical isolates of S. pneumoniae. This was associated with an eightfold to 256-fold increase in potency compared to ciprofloxacin $\left(\mathrm{MIC}_{90}: 64 \mu \mathrm{g} / \mathrm{mL}\right)$, levofloxacin $\left(\mathrm{MIC}_{90}: 64 \mu \mathrm{g} / \mathrm{mL}\right)$, moxifloxacin $\left(\mathrm{MIC}_{90}\right.$ : $16 \mu \mathrm{g} / \mathrm{mL}$ ), and gemifloxacin $\left(\mathrm{MIC}_{90}: 2 \mu \mathrm{g} / \mathrm{mL}\right){ }^{14}$

In another in vitro study, ${ }^{6}$ the activity of JNJ-Q2 against S. pneumoniae isolates from patients with CABP was compared against the activities of several other agents, including moxifloxacin, levofloxacin, ciprofloxacin, penicillin, ceftriaxone, cefuroxime, azithromycin, clindamycin, tetracycline, linezolid, and vancomycin. JNJ-Q2 was the most active agent tested against 2,137 isolates of $S$. pneumoniae, including isolates that were resistant to other fluoroquinolones. JNJ-Q2 had an associated $\mathrm{MIC}_{90}$ of $0.015 \mu \mathrm{g} / \mathrm{mL}$. All but one isolates (pneumococcus collected from Israel) were associated with elevated MICs of moxifloxacin [ $>8 \mu \mathrm{g} / \mathrm{mL}$ ], levofloxacin [ $>4 \mu \mathrm{g} / \mathrm{mL}]$, and ciprofloxacin [ $>4 \mu \mathrm{g} / \mathrm{mL}])$. Consequently, JNJ-Q2 was associated with 128-, 64-, and 16-fold higher potency than ciprofloxacin, levofloxacin, and moxifloxacin, respectively. For DRSP isolates, JNJ-Q2 had an elevated $\mathrm{MIC}_{90}(0.25 \mu \mathrm{g} / \mathrm{mL})$ compared to non-DRSP isolates. However, JNJ-Q2 remained the most active agent compared to other fluoroquinolones, whereby $\mathrm{MIC}_{90}$ values were eightfold, more than fourfold, and more than fourfold for moxifloxacin, levofloxacin, and ciprofloxacin, respectively. $\mathrm{MIC}_{90}$ values were slightly lower in this study, ${ }^{6}$ which is thought to be related to less number of ciprofloxacin-resistant strains.

\section{Potency against Haemophilus influenzae/Moraxella catarrhalis}

JNJ-Q2 displayed excellent in vitro activity against the respiratory pathogens Haemophilus influenzae and Moraxella catarrhalis. JNJ-Q2 had the same $\mathrm{MIC}_{90}(0.015 \mu \mathrm{g} / \mathrm{mL})$ against $H$. influenzae as ciprofloxacin; however, it had a lower $\mathrm{MIC}_{90}$ compared to moxifloxacin $(0.03 \mu \mathrm{g} / \mathrm{mL})$. Furthermore, when evaluated against $M$. catarrhalis, JNJ-Q2 had the lowest $\mathrm{MIC}_{90}(\leq 0.015 \mu \mathrm{g} / \mathrm{mL})$ compared to ciprofloxacin $(0.06 \mu \mathrm{g} / \mathrm{mL})$ and moxifloxacin $(0.06 \mu \mathrm{g} / \mathrm{mL}){ }^{14}$

\section{Resistance}

With the widespread use of fluoroquinolones, resistance to these agents has become an increasing concern. Resistance to fluoroquinolones among $S$. pneumoniae remains low in the US ( $<2 \%$ for levofloxacin and moxifloxacin); however, among $S$. aureus and some Gram-negative species, resistance is high and may limit the utility of currently approved fluoroquinolones. ${ }^{11,12}$ Fluoroquinolone resistance can arise through mutations of genes encoding enzymes. This results in the loss of affinity of the fluoroquinolone to its binding site, increase in the number of efflux pumps actively removing the fluoroquinolone, and modification of the permeability of the outer membrane in Gram-negative bacteria by closing the porin channels. ${ }^{4,14,21}$ Ciprofloxacin resistance in staphylococci may be attributable to its disproportionate affinity for DNA topoisomerases II and IV and the selection of efflux-based resistance. ${ }^{26-28}$ In vitro studies ${ }^{14,15,25}$ have evaluated the rate or frequency of spontaneous resistance to JNJ-Q2. Data from these studies suggest that JNJ-Q2 has a lower potential for development of resistance in $S$. pneumoniae and $S$. aureus compared to other fluoroquinolones. As mentioned previously, it is believed that this lower potential for resistance might be due to JNJ-Q2's improved balance in the potencies of inhibition of both DNA topoisomerase targets.

\section{Pharmacokinetics}

JNJ-Q2 has been studied as both an oral and intravenous (IV) dosage form similar to other fluoroquinolones. ${ }^{29}$ A comparison between the pharmacokinetic parameters of several common fluoroquinolone antibiotics and JNJ-Q2 is summarized in Table 2. The absolute oral bioavailability of JNJ-Q2 is $\sim 65 \%$, which is similar to that of ciprofloxacin, but far less than that of levofloxacin. ${ }^{29}$ To account for the bioavailability difference, pharmacokinetic studies of JNJ-Q2 were conducted using a $250 \mathrm{mg}$ oral dose compared to various IV doses. Single-dose studies ${ }^{29}$ with IV doses ranging from $15 \mathrm{mg}$ to $150 \mathrm{mg}$ revealed a dose-proportional change in area under the curve (AUC); however, there was a lessthan-proportional change in the maximum concentration that the drug achieved after dosing $\left(C_{\max }\right.$ value). The $250 \mathrm{mg}$ oral dose displayed a similar pharmacokinetic profile as the $150 \mathrm{mg}$ IV dosing. After one-time IV dosing of JNJ-Q2, a short distribution phase and extended terminal elimination phase were observed. In contrast, the oral formulation was observed to be monophasic, undergoing distribution and elimination simultaneously.

Multiple-dose studies ${ }^{29}$ in healthy volunteers receiving JNJ-Q2 $150 \mathrm{mg}$ parenterally twice daily revealed a half-life from 17.9 hours to 19.5 hours, steady state clearance ranging from $5.66 \mathrm{~L} / \mathrm{h}$ to $6.79 \mathrm{~L} / \mathrm{h}$, and a volume of distribution ranging from $120.9 \mathrm{~L}$ to $146.7 \mathrm{~L}$. Considering the half-life of $\sim 20$ hours, steady state concentrations were achieved on Day 4 of therapy. There is limited renal clearance of JNJ-Q2, 
Table 2 Comparison of pharmacokinetic parameters of selected fluoroquinolones

\begin{tabular}{|c|c|c|c|c|c|c|c|c|}
\hline \multirow[t]{2}{*}{ Single dose } & \multicolumn{2}{|c|}{ Ciprofloxacin ${ }^{43-45}$} & \multicolumn{2}{|c|}{ Levofloxacin ${ }^{46}$} & \multicolumn{2}{|c|}{ Moxifloxacin ${ }^{30,47}$} & \multicolumn{2}{|l|}{ JNJ-Q2 ${ }^{29}$} \\
\hline & $\begin{array}{l}500 \mathrm{mg}, \\
\text { PO }\end{array}$ & $\begin{array}{l}400 \mathrm{mg} \text {, } \\
\text { IV }\end{array}$ & $\begin{array}{l}500 \mathrm{mg}, \\
\text { PO }\end{array}$ & $\begin{array}{l}500 \mathrm{mg}, \\
\text { IV }\end{array}$ & $\begin{array}{l}400 \mathrm{mg}, \\
\text { PO }\end{array}$ & $\begin{array}{l}400 \mathrm{mg}, \\
\text { IV }\end{array}$ & $\begin{array}{l}250 \mathrm{mg}, \\
\text { PO }\end{array}$ & $\begin{array}{l}\text { I50 mg, } \\
\text { IV }\end{array}$ \\
\hline$C_{\max }(\mathrm{mg} / \mathrm{L})$ & 2.26 & 4.5 & 5.2 & 6.3 & 2.5 & 3.6 & 2.18 & 1.65 \\
\hline$t_{\max }$ (minutes) & 80 & - & 78 & - & 120 & - & 106 & - \\
\hline$V_{d}(\mathrm{~L} / \mathrm{kg})$ & 3.76 & - & 1.3 & 1.2 & 3.1 & 2.1 & $153.2^{\mathrm{a}}$ & $147.8^{\mathrm{a}}$ \\
\hline $\mathrm{CL}(\mathrm{L} / \mathrm{h})$ & 54.5 & 34.4 & 10.5 & 9.4 & 11.6 & 11.6 & 7.55 & 6.22 \\
\hline $\mathrm{AUC}_{24}(\mathrm{mg} / \mathrm{L} \cdot \mathrm{h})$ & 10 & 12 & 47.7 & 55.3 & 29.8 & 34.6 & 26.2 & 18.3 \\
\hline$t_{1 / 2}$ (hours) & 3.69 & 3.4 & 6.5 & 6.6 & 15.6 & 15.4 & 14.4 & 16.7 \\
\hline$F(\%)$ & 55.6 & - & $99^{b}$ & - & 86 & - & 65 & - \\
\hline Multidose & $\begin{array}{l}500 \mathrm{mg}, \\
\mathrm{PO}, \mathrm{q} / 2 \mathrm{~h}\end{array}$ & $\begin{array}{l}400 \text { mg, } \\
\text { IV, q8h }\end{array}$ & $\begin{array}{l}500 \mathrm{mg}, \\
\mathrm{PO}, \mathrm{q} 24 \mathrm{~h}\end{array}$ & $\begin{array}{l}500 \mathrm{mg}, \\
\text { IV, q24h }\end{array}$ & $\begin{array}{l}400 \mathrm{mg}, \\
\text { PO, q24h }\end{array}$ & $\begin{array}{l}400 \mathrm{mg} \\
\text { IV, q24h }\end{array}$ & $\begin{array}{l}250 \mathrm{mg}, \\
\mathrm{PO}, \mathrm{q} / 2 \mathrm{~h}\end{array}$ & $\begin{array}{l}\text { I50 mg, } \\
\text { IV, q I } 2 \mathrm{~h}\end{array}$ \\
\hline$C_{\max }(\mathrm{mg} / \mathrm{L})$ & 3.5 & 4.6 & 5.7 & 6.4 & 4.5 & - & - & 2.89 \\
\hline$t_{\max }$ (minutes) & 60 & - & 66 & - & - & - & - & - \\
\hline$V_{d}(L / k g)$ & - & - & 1.37 & 1.22 & - & - & - & 186.6 \\
\hline $\mathrm{CL}(\mathrm{L} / \mathrm{h})$ & 29 & 32 & 10.5 & 9.5 & - & - & - & 6.79 \\
\hline $\mathrm{AUC}_{24}(\mathrm{mg} / \mathrm{L} \cdot \mathrm{h})$ & 13.9 & 12.9 & 47.5 & 64.6 & 48 & - & - & - \\
\hline$t_{1 / 2}$ (hours) & 4.7 & 3.5 & 6.8 & 6.8 & 12 & - & - & 19.5 \\
\hline
\end{tabular}

Notes: aReported in liters. 'Levofloxacin data on file (Ortho-McNeil-Janssen Pharmaceuticals, Inc, Raritan, NJ, USA, 2008). - indicates there was no data for these fields. Abbreviations: $\mathrm{AUC}$, area under the curve; $\mathrm{CL}$, clearance; $C_{\max }$, maximum concentration of the drug achieved after dosing; IV, intravenous; $\mathrm{PO}$, oral; $\mathrm{q} 8 \mathrm{~h} / \mathrm{q} / 2 \mathrm{~h} / \mathrm{q} 24 \mathrm{~h}$, every 8 hours/ $/ 2$ hours/ 24 hours, respectively; $t_{1 / 2}$, terminal half-life; $t_{\max }$, peak time; $V_{\mathrm{d}}$, volume of distribution; $F$, bioavailability.

with only $12 \%$ of active drug being excreted in the urine and $23 \%$ excreted as minor metabolites. Due to the limited renal elimination, it is not expected that JNJ-Q2 will require dosage adjustment in patients with renal dysfunction. In addition, this may also limit the utility of JNJ-Q2 in the treatment of urinary tract infections because its urinary excretion is similar to that of moxifloxacin. Accumulation does occur after repeat IV doses of JNJ-Q2, with increases of $63 \%$ and $36 \%$ in $\mathrm{AUC}_{0-12}$ and $C_{\max }$, respectively. This degree of accumulation appears to be similar to that of moxifloxacin, but there can be substantial interpatient variability as evidenced by moxifloxacin pharmacokinetic studies that report accumulation ranging from $10 \%$ to $60 \%{ }^{30}$ Distribution studies have demonstrated significant JNJ-Q2 penetration into epithelial lining fluid (ELF) and alveolar macrophages (AMs). ${ }^{29} \mathrm{JNJ}$ Q2 concentrations in patients receiving $250 \mathrm{mg}$ orally twice daily were 50 times greater in ELF and 150 times greater in AMs compared to concentrations in serum. These ELF and AM concentrations are higher than those achieved with some other fluoroquinolones ${ }^{31}$; however, the clinical relevance of this finding remains to be seen. Overall, the pharmacokinetic profile of JNJ-Q2 is consistent with those of other fluoroquinolones, and these data have provided a basis for evaluating oral and parenteral regimens in clinical trials.

\section{Pharmacodynamics}

JNJ-Q2 is considered to be bactericidal against MSSA, MRSA, as well as $S$. pneumoniae. ${ }^{25}$ Animal model studies have provided some insight into the activity of JNJ-Q2.
JNJ-Q2 is significantly more active against MRSA than ciprofloxacin and moxifloxacin in septicemia, pneumonia, and skin infection models. ${ }^{25}$ Studies evaluating the pharmacodynamic targets of JNJ-Q2 are limited; however, the pharmacodynamic profile is expected to be similar to those of other fluoroquinolones. The pharmacodynamic profiles of fluoroquinolones are best described by AUC/MIC or $C_{\max }$ ' MIC, but there is a growing need to evaluate these targets with individual fluoroquinolones and specific pathogens. ${ }^{32}$ Further studies are needed to better define the appropriate pharmacodynamic profile for JNJ-Q2 in humans.

\section{Clinical efficacy Animal models}

A murine mode ${ }^{25}$ evaluated the in vivo potency of JNJ-Q2 in septicemia and established skin infections with MSSA and MRSA. In the septicemia model, JNJ-Q2 demonstrated similar activity to moxifloxacin against MSSA, in addition to showing superior activity relative to vancomycin against MRSA. In both MRSA skin infection models, treatment with JNJ-Q2 was associated with better responses (reductions in bacterial titers) per dose compared to ciprofloxacin, moxifloxacin, linezolid, and vancomycin. ${ }^{25}$

The in vivo efficacy of JNJ-Q2 against $S$. pneumoniae was assessed in a murine lower respiratory tract infection model. JNJ-Q2 was two- and tenfold more active than moxifloxacin administered by the oral and subcutaneous routes, respectively. Consequently, JNJ-Q2 was associated with statistically superior efficacy compared to moxifloxacin. Furthermore, 
JNJ-Q2 displayed similar activity compared to gemifloxacin when administered orally or subcutaneously. ${ }^{25}$

\section{Clinical trials}

Phase II clinical trials evaluating the safety and clinical efficacy of JNJ-Q2 in the setting of ABSSSI and CABP have been completed. ${ }^{5,17}$

Covington et $\mathrm{al}^{5}$ conducted a Phase II, randomized, double-blind, multicenter study in 18 sites in the US. Patients were randomly assigned to receive JNJ-Q2 (250 mg orally every 12 hours) or linezolid (600 mg orally every 12 hours) for 7-14 days for the treatment of ABSSSI. ${ }^{5}$ Subjects were stratified by infection type (wound infection, cellulitis, or severe abscess) and fever $\left(\geq 38^{\circ} \mathrm{C}\right)$ upon enrollment. Adult males and nonpregnant, nonlactating females with at least one systemic sign of infection and at least three of the following signs were included in the study: purulent drainage, erythema, fluctuation, heat/localized warmth, or pain/tenderness. Patients with prosthetic materials or foreign bodies, decubitus or diabetic foot ulcers, septic arthritis, gangrene, burns, deep venous thrombi, or severely compromised immune systems were excluded.

Among the 161 patients enrolled in the study, ${ }^{5}$ baseline characteristics were similar between treatment groups. The mean age was 36.9 years, and the majority of patients were white $(79.5 \%)$ males $(64.6 \%)$. Infection types in both the JNJ-Q2 and linezolid groups were quite similar, with approximately one-third of patients with abscesses, wound infections, and cellulitis. The mean and median lesion sizes for the study sample were $266 \mathrm{~cm}^{2}$ and $162 \mathrm{~cm}^{2}$, respectively. The overall rate of fever across both groups was $4.3 \%$, and $75 \%(121 / 161)$ of patients had a pathogen identified from the infection site. MRSA was identified in 45.8\% (38/83) and $41 \%(32 / 78)$ of patients treated with JNJ-Q2 and linezolid, respectively. All Gram-positive pathogens isolated at baseline were susceptible to JNJ-Q2 and linezolid.

Early clinical response, defined as the composite end point of stable temperature $<38^{\circ} \mathrm{C}$ and cessation of spread of the primary infection site 36-84 hours after the first dose of study medication was achieved in 74.7\% (62/83) and $79.5 \%(62 / 78)$ of patients treated with JNJ-Q2 and linezolid, respectively ${ }^{5}$ (odds ratio [OR]: $0.76 ; 95 \%$ confidence interval [CI]: 0.36, 1.59; $P=0.087)$. Statistically, JNJ-Q2 did not meet the criterion for the $15 \%$ noninferiority margin because the lower limit of the CI was not greater than the noninferiority limit of 0.469 . Prespecified clinical cure rates 2-14 days after treatment completion were similar between the groups (83.1\% for JNJ-Q2 vs 82.1\% for linezolid; OR: 1.06; CI:
$0.47,2.42 ; P=0.013)$. In a post hoc analysis, treatment response with JNJ-Q2 was noninferior to linezolid $(61.4 \%$ vs $57.7 \%$, respectively; OR: 1.14 ; CI: $0.6,2.15 ; P=0.024)$ based on the 2010 FDA guidance on treatment success in ABSSSI, which differed from the statistical analysis plan of the study. ${ }^{5}$ The FDA criteria assess cessation of spread or reduction in lesion size and temperature $<37.7^{\circ} \mathrm{C}$ at 48-72 hours. For patients with MRSA, the clinical cure rate was higher at Day 8 for JNJ-Q2 $(50.0 \%, 18 / 36)$ than for linezolid $(44.8 \%, 13 / 29)$, and the rate of clinical cure at short-term follow-up was $80.6 \%$ and $86.2 \%$ for JNJ-Q2 and linezolid, respectively. ${ }^{5}$

Covington et $\mathrm{al}^{17}$ conducted a second Phase II, randomized, double-blind, multicenter study evaluating the efficacy of JNJ-Q2 (150 mg IV every 12 hours, followed by $250 \mathrm{mg}$ orally every 12 hours), vs that of moxifloxacin (400 mg IV or orally every 24 hours) for the treatment of CABP. Hospitalized adults with a pneumonia severity index score of $\geq$ II and at least three signs or symptoms of CABP (cough, dyspnea/tachypnea, chest pain, fever/hypothermia, or pulmonary consolidation) were included. Additionally, baseline sputum cultures with a positive Gram's stain and a chest X-ray (CXR) showing infiltrates were required for inclusion. The study was conducted in 60 centers in North America, Eastern Europe, and Latin America, and a total of 896 patients were screened for eligibility, of which 32 were randomized. Of note, the study was terminated early due to slow enrollment. The study was designed to enroll 120 subjects to ensure ability to detect noninferiority of JNJ-Q2 to moxifloxacin; however, this was not possible due to the strict inclusion criteria. The slow enrollment was mainly due to prior antibiotic use or failure to meet requirements for CXR or sputum production with positive Gram's stain. Prior antibiotic use led to the exclusion of $25.5 \%(220 / 864)$ of patients, while $17 \%$ (147/864) of patients were excluded due to $\mathrm{CXR} /$ sputum requirement. Among patients enrolled in the study, ${ }^{17} 87.5 \%$ (28/32) had an identified pathogen, with $S$. pneumoniae being the most common isolate $(84 \%)$. All $S$. pneumoniae isolates were susceptible to JNJ-Q2 and moxifloxacin. The authors did not report other baseline characteristics for included patients. Clinical cure was achieved in $87.5 \%(14 / 16)$ and $81.3 \%(13 / 16)$ of patients treated with JNJ-Q2 and moxifloxacin, respectively (OR: 1.66; CI: 0.23, 11.75). Because of the small sample size, there was insufficient power to show noninferiority for clinical test of cure. Criteria for early response at Day 4, which was a combination of clinically stable vital signs and symptom success, were met in $56.3 \%$ (nine out of 16 ) and $43.8 \%$ (seven out of 16 ) 
subjects treated with JNJ-Q2 and moxifloxacin, respectively (OR: 1.59; CI: 0.27, 9.42). ${ }^{17}$

\section{Safety}

The safety of JNJ-Q2 has been evaluated in multiple Phase I and II clinical studies. ${ }^{5,17,29,33}$ Overall, these studies demonstrate that JNJ-Q2 is generally well tolerated.

In a multiple ascending-dose study, six participants received multiple doses of JNJ-Q2 $150 \mathrm{mg} \mathrm{IV}$, and seven adverse events were reported (contact dermatitis [1], infusion site extravasation [1], chest pain [1], increased heart rate [1], and phlebitis [3]). ${ }^{29}$ In the same study, a single participant experienced two episodes of asymptomatic hypoglycemia after being administered multiple doses of JNJ-Q2 $100 \mathrm{mg}$ IV. Of note, this participant had preexisting hypoglycemia prior to receiving JNJ-Q2. Further analysis of all glucose values for study participants revealed no pattern suggestive of impaired glucose homeostasis with JNJ-Q2. In a sequential single ascending-dose study ${ }^{29}$ that also evaluated absolute oral bioavailability, 14 participants received JNJ-Q2 $250 \mathrm{mg}$ orally. The most common adverse event reported with the oral formulation was nausea $(14.3 \%) .{ }^{29}$ In a single-center, repeat oral dose lung penetration study, three out of six (50\%) participants reported four adverse events after receiving JNJ-Q2 $250 \mathrm{mg}$ orally. The most significant event involved elevations in alanine aminotransferase (ALT), aspartate aminotransferase, and $\gamma$-glutamyl transferase after 4 days of JNJ-Q2 administration. It is important to note, however, that the participant was asymptomatic, concomitant elevations in bilirubin were not observed, and the condition was completely reversible.

The cardiovascular safety of JNJ-Q2 was evaluated in a comprehensive set of nonclinical and clinical studies. ${ }^{33}$ The effect of JNJ-Q2 on different cardiovascular parameters was compared with the effects of moxifloxacin, sparfloxacin, and ofloxacin. JNJ-Q2 and comparators were evaluated in various models/test systems, including human ether-ago-go-related gene ( $h E R G$ )-transfected human embryonic kidney cells, sodium channel-transfected Chinese hamster ovary cells, guinea pig right atria, and arterially perfused rabbit left ventricular wedge preparations, in in vivo studies in guinea pigs and dogs, as well as in a thorough QT study in humans. ${ }^{33}$ The study evaluating the effect of JNJQ2 on the corrected QT (QTc) interval in humans was a randomized, double-blind, placebo- and positive-controlled, four-period crossover study. ${ }^{33}$ In each treatment period, subjects received JNJ-Q2 $250 \mathrm{mg}$ twice daily or $500 \mathrm{mg}$ daily for four consecutive days to achieve steady state; placebo or a single dose of moxifloxacin $400 \mathrm{mg}$ served as the positive control. Mean differences in heart rate from predose baseline were minor and not clinically significant. Similarly, JNJ-Q2 had no appreciable effects on the PR and QRS intervals. Both JNJ-Q2 treatment regimens increased the QTc interval by $12 \mathrm{~ms}$ from baseline, but the observed changes following each dose were less than those observed following a single dose of moxifloxacin $400 \mathrm{mg}$. Overall, the study ${ }^{33}$ concluded that JNJ-Q2 causes a less pronounced increase in QTc interval compared with moxifloxacin and sparfloxacin.

Covington et $\mathrm{al}^{5}$ evaluated the safety and efficacy of JNJ-Q2 vs linezolid for the treatment of ABSSSI in a Phase II trial. Overall, the incidence of serious adverse events in this study ${ }^{5}$ was low, and the majority of adverse events were mild or moderate in severity. Rates of adverse events reported between groups were similar, except for nausea $(22.9 \%$ vs $11.4 \%$ ) and vomiting (12\% vs $6.3 \%$ ), which occurred more frequently in the JNJ-Q2 group. Approximately $80 \%$ of patients who experienced nausea and vomiting with JNJQ2 had resolution of symptoms within the first 2 days of therapy, and no patients discontinued the study drug due to these symptoms. Patients were not specifically instructed to take the study medication with food, which is being done in subsequent studies to potentially lower the frequency of gastrointestinal events. The incidence of diarrhea was similar between JNJ-Q2 (14.5\%) and linezolid (16.5\%). All patients with diarrhea were evaluated for Clostridium difficile infection, and no cases were identified. Transient elevations in ALT at least 1.5 times the upper limit of normal were observed in $<10 \%$ of patients and were similar between groups $(8.4 \%$ for JNJ-Q2 vs $8.9 \%$ for linezolid). One patient treated with JNJ-Q2 had an asymptomatic elevation of ALT to $875 \mathrm{IU} / \mathrm{L}$ without a concomitant increase in bilirubin, which resolved within 30 days. Rash was reported in $<5 \%$ of patients and was similar between groups. There were no visual disturbances, seizures, or hematologic abnormalities reported in this study. ${ }^{5}$ Additionally, no patients receiving JNJ-Q2 experienced dysglycemia or changes in the QTc interval. ${ }^{5}$

In the CABP Phase II clinical trial conducted by Covington et al, ${ }^{17}$ adverse events were comparable between JNJ-Q2 and moxifloxacin, with the exception of nausea and vomiting. Unlike the previous study, ${ }^{5}$ nausea and vomiting were observed more frequently in patients treated with moxifloxacin than with JNJ-Q2. ${ }^{17}$ Unfortunately, detailed information regarding the incidence, relationship to study medication, and severity of adverse events reported in this study are not available. 


\section{Discussion}

Antibiotic resistance continues to be a major concern, and new antibiotics are essential for the appropriate treatment of infections caused by resistant bacteria. In fact, the World Health Organization has identified antimicrobial resistance as one of the three greatest threats to human health. The CDC published a report in 2013 to increase awareness regarding the threat that antibiotic resistance poses and the immediate actions needed to be taken. ${ }^{3}$ In this report, numerous bacteria were identified as potential threats, including MRSA and DRSP, which comprise the topics of this review.

Although once considered a nosocomial pathogen, MRSA is now a common cause of community-acquired infections, most notably ABSSSI. ${ }^{34}$ In one study ${ }^{35}$ including 422 patients seen in the emergency department for purulent soft tissue infections, MRSA was the most common causative pathogen, isolated from $59 \%$ of patients. Furthermore, the rate of hospital admissions for ABSSSI has increased in recent years, which may be due to the emergence of community-acquired MRSA. ${ }^{36,37}$ The increased incidence of community-acquired MRSA is concerning, as the rates of resistance to erythromycin, clindamycin, tetracycline, and sulfamethoxazoletrimethoprim have increased over the years. ${ }^{9}$ Similarly, resistance to vancomycin, daptomycin, and linezolid has surfaced. ${ }^{38-40} S$. pneumoniae is the most common bacterial pathogen associated with CABP in hospitalized adults in the US. ${ }^{41}$ Respiratory fluoroquinolones, including levofloxacin and moxifloxacin, are currently recommended for the empiric treatment of CABP in adults. ${ }^{10}$ Though the incidence of resistance to levofloxacin and moxifloxacin is extremely low, DRSP is still a potential concern. Unfortunately, currently available fluoroquinolones do not have reliable activity against MRSA and therefore are not recommended for empiric treatment of ABSSSI. ${ }^{9}$ Furthermore, the potential for the emergence of levofloxacin- and moxifloxacin-resistant S. pneumoniae is plausible and could limit them as options to empirically treat CABP.

JNJ-Q2 is a novel fluoroquinolone antibiotic with broadspectrum bactericidal activity, including excellent activity against MRSA and DRSP. ${ }^{5}$ Unlike other fluoroquinolones, JNJ-Q2 has an improved balance in the potencies of inhibition of DNA gyrase and DNA topoisomerase IV, which is thought to decrease the development of drug resistance. ${ }^{14,15,21}$ Multiple in vivo and in vitro studies ${ }^{6,14,15,17,23-25}$ demonstrate that JNJ-Q2 has excellent activity against a variety of Gram-positive and Gram-negative pathogens, including MSSA, MRSA, and fluoroquinolone-resistant $S$. pneumoniae. Specifically,
JNJ-Q2 had the highest potency among all agents tested against $S$. pneumoniae, $M$. catarrhalis, MSSA, MRSA, and beta-hemolytic streptococci. ${ }^{6,14,15,17,23-25}$ The available safety data suggest that JNJ-Q2 has a favorable safety profile. Clinical studies have demonstrated that JNJ-Q2 has adverse event rates similar to or less than those of other fluoroquinolones, notably QTc prolongation, dysglycemia, and photosensitivity reactions. ${ }^{5,17}$ However, further investigation will be needed to establish a robust safety profile for JNJ-Q2 in diverse patient populations.

The clinical efficacy of JNJ-Q2 in ABSSSI was compared to that of linezolid in a Phase II clinical trial, ${ }^{5}$ in which nearly half of the study participants at baseline had MRSA isolated from a wound culture. JNJ-Q2 did not meet criteria for noninferiority for the primary end point, early clinical response; however, a post hoc analysis based on new FDA guidance for evaluating agents in the treatment of ABSSSI revealed that JNJ-Q2 was noninferior to linezolid ( $61.4 \%$ vs $57.7 \% ; P=0.024) .^{5}$ This cited study demonstrated JNJ-Q2 could represent a valuable treatment option for patients with purulent ABSSSI, especially considering it is available in oral and IV formulations and would allow for sequential therapy similar to the oxazolidinones, linezolid and tedizolid. Unlike the oxazolidinones, however, concerns for hematological effects and drug interactions with serotonergic agents have not been associated with JNJ-Q2 and could potentially favor its use.

In a smaller Phase II clinical trial, ${ }^{17}$ the efficacy of JNJQ2 was compared to that of moxifloxacin in the treatment of CABP. Patients with a positive Gram-stain and evidence of pneumonia on CXR were randomized to receive moxifloxacin or JNJ-Q2. Overall, clinical cure was achieved in 14/16 $(87.5 \%)$ and 13/16 (81.3\%) subjects treated with JNJ-Q2 and moxifloxacin, respectively. ${ }^{17}$ Unfortunately, recruitment into the study was hindered due to strict inclusion criteria; thus there was insufficient power to show noninferiority for clinical test of cure. Despite its small sample size, the results of this study ${ }^{17}$ are encouraging.

In the context of ABSSSI and CABP, JNJ-Q2 appears to be a promising option and current data support continued development of this agent. JNJ-Q2 appears to have a more promising future in the management of ABSSSI given there are limited treatment options for MRSA with both oral and IV formulations available. On the other hand, JNJ-Q2 would serve more as an alternative option for CABP considering currently available fluoroquinolones (levofloxacin and moxifloxacin) are dosed once daily, have better bioavailability, and have limited resistance. 


\section{Conclusion}

JNJ-Q2 is a novel, broad-spectrum fluoroquinolone that has superior in vitro potency compared to other fluoroquinolones, particularly against quinolone-susceptible and -resistant S. aureus (including MRSA) and DRSP. In addition, in vitro studies show that JNJ-Q2 has a lower propensity for the development of resistance compared to other agents in the class. Considering its expanded spectrum of activity against these resistant Gram-positive pathogens and its low potential for resistance, JNJ-Q2 shows promise as an effective treatment option for ABSSSI and CABP. Furthermore, Phase II studies have demonstrated comparable efficacy results with JNJ-Q2, compared to linezolid for ABSSSI and to moxifloxacin for CABP, as well as a favorable safety profile. However, considering its early stage of development, the definitive role of JNJ-Q2 for these infections, as well as its safety profile, will be determined in future Phase III studies. Unfortunately, there are no Phase III studies currently enrolling patients at the time of this review, but the prospect of another oral agent with activity against MRSA and DRSP could improve the arsenal to combat infections caused by antibiotic-resistant bacteria in the future.

\section{Disclosure}

The authors report no conflicts of interest in this work.

\section{References}

1. Hong MC, Hsu DI, Bounthavong M. Ceftolozane/tazobactam: a novel antipseudomonal cephalosporin and beta-lactamase-inhibitor combination. Infect Drug Resist. 2013;6:215-223.

2. Mitra S, Saeed U, Havlichek DH, Stein GE. Profile of oritavancin and its potential in the treatment of acute bacterial skin structure infections. Infect Drug Resist. 2015;8:189-197.

3. CDC [webpage on the Internet]. Antibiotic Resistance Threats in the United States, 2013. Available from: http://www.cdc.gov/drugresistance/ threat-report-2013/. Accessed December 30, 2015.

4. Redgrave LS, Sutton SB, Webber MA, Piddock LJ. Fluoroquinolone resistance: mechanisms, impact on bacteria, and role in evolutionary success. Trends Microbiol. 2014;22(8):438-445.

5. Covington P, Davenport JM, Andrae D, et al. Randomized, double-blind, phase II, multicenter study evaluating the safety/tolerability and efficacy of JNJ-Q2, a novel fluoroquinolone, compared with linezolid for treatment of acute bacterial skin and skin structure infection. Antimicrob Agents Chemother. 2011;55(12):5790-5797.

6. Biedenbach DJ, Farrell DJ, Flamm RK, Liverman LC, McIntyre G, Jones RN. Activity of JNJ-Q2, a new fluoroquinolone, tested against contemporary pathogens isolated from patients with community-acquired bacterial pneumonia. Int J Antimicrob Agents. 2012;39(4):321-325.

7. Blumberg HM, Rimland D, Carroll DJ, Terry P, Wachsmuth IK. Rapid development of ciprofloxacin resistance in methicillin-susceptible andresistant Staphylococcus aureus. J Infect Dis. 1991;163(6):1279-1285.

8. Jones RN, Mendes RE, Sader HS. Ceftaroline activity against pathogens associated with complicated skin and skin structure infections: results from an international surveillance study. J Antimicrob Chemother. 2010;65(Suppl 4):iv17-iv31.
9. Stevens DL, Bisno AL, Chambers HF, et al. Practice guidelines for the diagnosis and management of skin and soft tissue infections: 2014 update by the infectious diseases society of America. Clin Infect Dis. 2014;59(2):147-159.

10. Mandell LA, Wunderink RG, Anzueto A, et al. Infectious Diseases Society of America/American Thoracic Society consensus guidelines on the management of community-acquired pneumonia in adults. Clin Infect Dis. 2007;44(Suppl 2):S27-S72.

11. Tomic V, Dowzicky MJ. Regional and global antimicrobial susceptibility among isolates of Streptococcus pneumoniae and Haemophilus influenzae collected as part of the Tigecycline Evaluation and Surveillance Trial (T.E.S.T.) from 2009 to 2012 and comparison with previous years of T.E.S.T. (2004-2008). Ann Clin Microbiol Antimicrob. 2014;13:52.

12. Jones RN, Sader HS, Mendes RE, Flamm RK. Update on antimicrobial susceptibility trends among Streptococcus pneumoniae in the United States: report of ceftaroline activity from the SENTRY Antimicrobial Surveillance Program (1998-2011). Diagn Microbiol Infect Dis. 2013;75(1):107-109.

13. Lee B, Boucher HW. Targeting antimicrobial-resistant bacterial respiratory tract pathogens: it is time to 'get smart'. Curr Opin Pulm Med. 2015;21(3):293-303.

14. Morrow BJ, He W, Amsler KM, et al. In vitro antibacterial activities of JNJ-Q2, a new broad-spectrum fluoroquinolone. Antimicrob Agents Chemother. 2010;54(5):1955-1964.

15. Morrow BJ, Abbanat D, Baum EZ, et al. Antistaphylococcal activities of the new fluoroquinolone JNJ-Q2. Antimicrob Agents Chemother. 2011;55(12):5512-5521.

16. Van Bambeke F. Renaissance of antibiotics against difficult infections: focus on oritavancin and new ketolides and quinolones. Ann Med. 2014;46(7):512-529.

17. Covington PS, Davenport JM, Andrae DA, et al. A Phase 2 study of the novel fluoroquinolone JNJ-Q2 in community-acquired bacterial pneumonia. J Antimicrob Chemother. 2013;68(11):2691-2693.

18. Owens RC Jr, Ambrose PG. Antimicrobial safety: focus on fluoroquinolones. Clin Infect Dis. 2005;41(Suppl 2):S144-S157.

19. Andriole VT. The quinolones: past, present, and future. Clin Infect Dis. 2005;41(Suppl 2):S113-S119.

20. Domagala JM. Structure-activity and structure-side-effect relationships for the quinolone antibacterials. J Antimicrob Chemother. 1994;33(4):685-706.

21. Karpiuk I, Tyski S. Looking for the new preparations for antibacterial therapy III. New antimicrobial agents from the quinolones group in clinical trials. Przegl Epidemiol. 2013;67(3):455-460, 557-461.

22. Zhao X, Xu C, Domagala J, Drlica K. DNA topoisomerase targets of the fluoroquinolones: a strategy for avoiding bacterial resistance. Proc Natl Acad Sci U S A. 1997;94(25):13991-13996.

23. Farrell DJ, Liverman LC, Biedenbach DJ, Flamm RK, Jones RN. Surveillance of JNJ-Q2 activity tested against Staphylococcus aureus and beta-hemolytic Streptococci as a component of the 2010 SENTRY Antimicrobial Surveillance Program. Diagn Microbiol Infect Dis. 2011;71(4):415-420.

24. Farrell DJ, Liverman LC, Biedenbach DJ, Jones RN. JNJ-Q2, a new fluoroquinolone with potent in vitro activity against Staphylococcus aureus, including methicillin- and fluoroquinolone-resistant strains. Antimicrob Agents Chemother. 2011;55(7):3631-3634.

25. Fernandez J, Hilliard JJ, Morrow BJ, et al. Efficacy of a new fluoroquinolone, JNJ-Q2, in murine models of Staphylococcus aureus and Streptococcus pneumoniae skin, respiratory, and systemic infections. Antimicrob Agents Chemother. 2011;55(12):5522-5528.

26. Kaatz GW, Moudgal VV, Seo SM. Identification and characterization of a novel efflux-related multidrug resistance phenotype in Staphylococcus aureus. J Antimicrob Chemother. 2002;50(6):833-838.

27. Drlica K, Malik M. Fluoroquinolones: action and resistance. Curr Top Med Chem. 2003;3(3):249-282. 
28. Firsov AA, Vostrov SN, Lubenko IY, Drlica K, Portnoy YA, Zinner SH. In vitro pharmacodynamic evaluation of the mutant selection window hypothesis using four fluoroquinolones against Staphylococcus aureus. Antimicrob Agents Chemother. 2003;47(5):1604-1613.

29. Davenport JM, Covington P, Gotfried M, et al. Summary of pharmacokinetics and tissue distribution of a broad-spectrum fluoroquinolone, JNJ-Q2. Clin Pharmacol Drug Dev. 2012;1(4):121-130.

30. Sullivan JT, Woodruff M, Lettieri J, et al. Pharmacokinetics of a oncedaily oral dose of moxifloxacin (Bay 12-8039), a new enantiomerically pure 8-methoxy quinolone. Antimicrob Agents Chemother. 1999;43(11):2793-2797.

31. Stass H, Dalhoff A, Kubitza D, Schuhly U. Pharmacokinetics, safety, and tolerability of ascending single doses of moxifloxacin, a new 8-methoxy quinolone, administered to healthy subjects. Antimicrob Agents Chemother. 1998;42(8):2060-2065.

32. Labreche MJ, Frei CR. Declining susceptibilities of gram-negative bacteria to the fluoroquinolones: effects on pharmacokinetics, pharmacodynamics, and clinical outcomes. Am J Health Syst Pharm. 2012;69(21):1863-1870.

33. Eichenbaum G, Pugsley MK, Gallacher DJ, et al. Role of mixed ion channel effects in the cardiovascular safety assessment of the novel anti-MRSA fluoroquinolone JNJ-Q2. Br J Pharmacol. 2012;166(5):1694-1707.

34. Corey GR, Stryjewski ME. New rules for clinical trials of patients with acute bacterial skin and skin-structure infections: do not let the perfect be the enemy of the good. Clin Infect Dis. 2011;52 (Suppl 7):S469-S476.

35. Moran GJ, Krishnadasan A, Gorwitz RJ, et al; EMERGEncy ID Net Study Group. Methicillin-resistant $S$. aureus infections among patients in the emergency department. $N$ Engl J Med. 2006;355(7):666-674.

36. Edelsberg J, Taneja C, Zervos M, et al. Trends in US hospital admissions for skin and soft tissue infections. Emerg Infect Dis. 2009;15(9):1516-1518.

37. Pallin DJ, Espinola JA, Leung DY, Hooper DC, Camargo CA Jr. Epidemiology of dermatitis and skin infections in United States physicians' offices, 1993-2005. Clin Infect Dis. 2009;49(6):901-907.
38. Centers for Disease Control and Prevention (CDC). Staphylococcus aureus resistant to vancomycin - United States, 2002. MMWR Morb Mortal Wkly Rep. 2002;51(26):565-567.

39. Skiest DJ. Treatment failure resulting from resistance of Staphylococcus aureus to daptomycin. J Clin Microbiol. 2006;44(2):655-656.

40. Sanchez Garcia M, De la Torre MA, Morales G, et al. Clinical outbreak of linezolid-resistant Staphylococcus aureus in an intensive care unit. JAMA. 2010;303(22):2260-2264.

41. Jain S, Self WH, Wunderink RG, et al; CDC EPIC Study Team. Community-acquired pneumonia requiring hospitalization among U.S. adults. $N$ Engl J Med. 2015;373(5):415-427.

42. Biedenbach DJ, Turner LL, Jones RN, Farrell DJ. Activity of JNJQ2, a novel fluoroquinolone, tested against Neisseria gonorrhoeae, including ciprofloxacin-resistant strains. Diagn Microbiol Infect Dis. 2012;74(2):204-206.

43. LeBel M, Vallee F, Bergeron MG. Tissue penetration of ciprofloxacin after single and multiple doses. Antimicrob Agents Chemother. 1986;29(3):501-505.

44. Gonzalez MA, Moranchel AH, Duran S, et al. Multiple-dose pharmacokinetics of ciprofloxacin administered intravenously to normal volunteers. Antimicrob Agents Chemother. 1985;28(2):235-239.

45. Sorgel F, Naber KG, Kinzig M, Mahr G, Muth P. Comparative pharmacokinetics of ciprofloxacin and temafloxacin in humans: a review. Am J Med. 1991;91(6A):51S-66S.

46. Holland M, Chien SC, Corrado M, Flor SC, Rogge MC. The pharmacokinetic profile of levofloxacin following once- or twice-daily $500 \mathrm{mg}$ administration. Poster presented at: Fifth International Symposium on New Quinolones; August 25-27, 1994; Singapore.

47. Stass H, Kubitza D. Pharmacokinetics and elimination of moxifloxacin after oral and intravenous administration in man. J Antimicrob Chemother. 1999;43(Suppl B):83-90.
Infection and Drug Resistance

\section{Publish your work in this journal}

Infection and Drug Resistance is an international, peer-reviewed openaccess journal that focuses on the optimal treatment of infection (bacterial, fungal and viral) and the development and institution of preventive strategies to minimize the development and spread of resistance. The journal is specifically concerned with the epidemiology of antibiotic

\section{Dovepress}

resistance and the mechanisms of resistance development and diffusion in both hospitals and the community. The manuscript management system is completely online and includes a very quick and fair peerreview system, which is all easy to use. Visit http://www.dovepress.com/ testimonials.php to read real quotes from published authors. 